\title{
Anticancer effects of gemcitabine are enhanced by co-administered iRGD peptide in murine pancreatic cancer models that overexpressed neuropilin-1
}

\author{
Y Akashi ${ }^{1}$, T Oda ${ }^{*}{ }^{1}$, Y Ohara ${ }^{1}$, R Miyamoto ${ }^{1}$, T Kurokawa ${ }^{1}$, S Hashimoto ${ }^{1}$, T Enomoto ${ }^{1}$, K Yamada $^{1}$, M Satake ${ }^{2}$ \\ and $\mathrm{N}$ Ohkohchi ${ }^{1}$ \\ ${ }^{1}$ Department of Surgery, Clinical Sciences, Graduate School of Comprehensive Human Sciences, University of Tsukuba, 1-1-1 \\ Tennoudai, Tsukuba, 305-8575 Ibaraki, Japan and '2Department of Diagnostic Radiology, Research Center for Innovative \\ Oncology, National Cancer Center Hospital East, Kashiwa, Chiba, Japan
}

Background: Impaired drug transport is an important factor that reduces the efficacy of anticancer agents against pancreatic cancer. Here, we report a novel combination chemotherapy using gemcitabine (GEM) and internalised-RGD (iRGD) peptide, which enhances tumour-specific drug penetration by binding neuropilin-1 (NRP1) receptor.

Methods: A total of five pancreatic cancer murine models (two cell line-based xenografts (CXs) and three tumour grafts (TGs)) were treated with either GEM $\left(100 \mathrm{mg} \mathrm{kg}^{-1}, \mathrm{q} 3 \mathrm{~d} \times 4\right)$ alone or GEM plus iRGD peptide $\left(8 \mu \mathrm{mol} \mathrm{kg}{ }^{-1}\right)$. Evaluation of NRP1 expression in xenografts and 48 clinical cancer specimens was performed by immunohistochemistry (IHC).

Results: We identified a subset of pancreatic cancer models that showed NRP1 overexpression sensitive to iRGD coadministration. Treatment with GEM plus IRGD peptide resulted in a significant tumour reduction compared with GEM monotherapy in CXs, but not remarkable in TGs. Potential targets of IRGD were characterised as cases showing NRP1 overexpression (IHC-2+/3+), and these accounted for $45.8 \%$ of the clinical specimens.

Conclusions: Internalised RGD peptide enhances the effects of co-administered drugs in pancreatic cancer models, its efficacy is however only appreciable in those employing cell lines. Therefore, the clinical application needs to be given careful consideration.

Pancreatic cancer is one of the most intractable human malignancies, and the median survival of patients receiving the current standard therapeutic regimen with gemcitabine (GEM) is $<6$ months (Burris et al, 1997; Tempero et al, 2003). Insufficient therapeutic efficacy of anticancer drugs for solid tumours may be explained by the limited penetration of drugs into the cancer tissue (Ruoslahti et al, 2010). In fact, intravenously injected drugs are known to penetrate only a few cell diameters from blood vessels into the extravascular tumour tissue (Jain, 1999; Heldin et al, 2004). Therefore, a strategy for inducing drug hyperpermeability from tumour vessels might improve drug delivery to solid tumours.
Recently, a peptide known as iRGD (internalised-RGD) has developed (Sugahara et al, 2009, 2010). This peptide induces tumour-specific vascular hyperpermeability by binding to $\alpha \mathrm{v}$ integrins that are specifically expressed on the endothelium of the tumour or angiogenic vessels. Co-administration of this peptide has been shown to markedly enhance the intratumoral accumulation of various agents with a wide range of molecular sizes in comparison with single administration, including doxorubicin (580 Da, 7-fold), dextran (1300-10000 Da, 3- to 5-fold), Evans blue dye (66 $000 \mathrm{Da}, 2$ - to 4-fold), trastuzumab (148 $000 \mathrm{Da}$, 40-fold), doxorubicin-liposome (Doxil, Janssen Pharmaceutica, 
Beerse, Belgium) (120 nm, 14-fold), and nab-paclitaxel (abraxane; Abraxis BioScience, Los Angeles, CA, USA) (130 nm, 9- to 12-fold), in mouse breast or prostate cancer models (Sugahara et al, 2010).

Activation of circulating iRGD peptide requires interaction with neuropilin-1 (NRP1) (Sugahara et al, 2009). Intravenously administered iRGD peptide initially accumulates in tumour vessels by binding to endothelial $\alpha \mathrm{v}$ integrin, and then its $\mathrm{C}$-terminus is cleaved to expose an arginine or lysine residue ( $=\mathrm{C}$-end rule (CendR) motif), before drug penetration deeply into the parenchyma of solid tumours occurs through interaction with NRP1 (Sugahara et al, 2009; Teesalu et al, 2009). Neuropilin-1 is a multifunctional non-tyrosine kinase receptor that binds to class 3 semaphorins and the vascular endothelial growth factor (VEGF) family, playing essential roles in mediating axonal guidance and angiogenesis (Soker et al, 1996; Kolodkin et al, 1997). It is reportedly correlated with tumour progression and/or poor prognosis in various cancers, including pancreatic cancer (Parikh et al, 2003; Hansel et al, 2004; Li et al, 2004; Fukahi et al, 2004; Müller et al, 2007). However, as the clinical sample sizes evaluated were limited, that is, only 10 cases or less, the expression profile and prognostic significance of NRP1 in pancreatic cancer has remained unclear.

The characteristics of iRGD peptide in solid cancers seem to warrant further investigation, especially in those with a dismal prognosis such as pancreatic cancer. However, there have been no comprehensive in vivo studies using pancreatic cancer models, and the effects of iRGD peptide used in combination with GEM, a key drug employed worldwide for treatment of pancreatic cancer, have not been tested. In the present study, therefore, we examined the effectiveness of iRGD peptide for boosting drug accumulation in eight different mouse pancreatic cancer xenograft models. The anticancer booster effect of combination therapy with GEM plus iRGD peptide was evaluated with reference to in vivo experiments and analysis of NRP1 expression in clinical cases of pancreatic cancer.

MATERIALS AND METHODS

Drugs. The iRGD peptide (Sequence; CRGDKGPDC, disulphide bridge; C1-C9, Purity; > 95\%) we used for this study was delivered from a contract manufacturing company (American Peptide Company, Sunnyvale, CA, USA). A small quantity of the original iRGD peptide and control peptide (iRGE; CRGEKGPDC) were kindly provided by Drs Sugahara and Ruoslahti (UCSB, University of California, Santa Barbara), and the pharmaceutical power of our peptide was confirmed to be equivalent (data not shown). The other drugs purchased were Evans blue dye (MP Biomedicals, Santa Ana, CA, USA), dextran Alexa fluor 488 (D-22910, 10000 Da MW; Molecular Probes, Eugene, OR, USA), and GEM (Gemzar; Eli Lilly, Indianapolis, IN, USA).

Cell lines and cell line-based models (= cell xenografts). The human pancreatic cancer cell lines AsPC-1 (CRL-1682), BxPC-3 (CRL-1687), and Capan-1 (HTB-79) were purchased from ATCC (Manassas, VA, USA). MIA PaCa-2 (JCRB0070) and SUIT-2 (JCRB1094) were purchased from JCRB Cell Bank (Osaka, Japan). AsPC-1, BxPC-3, and Capan-1 were cultured in RPMI-1640 medium (Sigma-Aldrich, MO, USA) with $10 \%$ fetal bovine serum. SUIT-2 and MIA PaCa-2 were cultured in Dulbecco's Modified Eagle Medium (DMEM; Sigma-Aldrich) with 5\% and 10\% fetal bovine serum. CXs were created by injecting these human pancreatic cancer cells $\left(5 \times 10^{6}\right.$ cells $)$ into the dorsal subcutaneous space of C.B-17/Icrscid/scid mice (female, 6 to 8 weeks old, CLEA Japan, Tokyo, Japan).

Transplant models using surgically removed tumour fragments (= tumor grafts). Pancreatic tumour grafts (TGs) were established by transplantation of fresh surgically resected tissue fragments of human pancreatic cancer. Samples were obtained under sterile conditions, avoiding apparently necrotic tissue and areas of normal pancreatic parenchyma. The samples were cut into $2 \times 2 \times 2 \mathrm{~mm}$ pieces, placed immediately in normal saline solution, and cooled on ice until implantation (usually within $30 \mathrm{~min}$ to $1 \mathrm{~h}$ ). All human samples employed in the experiments were harvested after informed consent in documents, and the establishment and the experimental use of 'tumour grafts' were approved by laboratory animal resource centre at University of Tsukuba. More details about the methods and characteristics of TGs are described in our previous report (Akashi et al, 2013).

In vivo systemic permeability assay. Tumour-bearing mice were injected intravenously with $100 \mu \mathrm{l}$ of PBS containing either $1 \%$ Evans blue dye or $0.3 \mathrm{mg}$ of dextran. Internalised-RGD peptide $\left(100 \mu \mathrm{l}, 12 \mu \mathrm{mol} \mathrm{kg}^{-1}\right)$ or PBS was also injected via the tail vein $10 \mathrm{~min}$ beforehand. After $30 \mathrm{~min}$ of circulation, the mice were perfused with PBS containing 1\% BSA, and their organs were collected. For Evans blue quantification, the dye was extracted from whole tumour tissue collected from each mice in $N$, $\mathrm{N}$-dimethyl formamide (Sigma-Aldrich) for $24 \mathrm{~h}$ at $37^{\circ} \mathrm{C}$ and quantified by measuring the absorbance at $600 \mathrm{~nm}$ with a spectrophotometer and the measured results were normalised by the tumour weight (Sugahara et al, 2010). Harvest tissues after dextran administration group were immediately fixed with $4 \%$ paraformaldehyde for $24 \mathrm{~h}$, frozen in optimum cutting temperature (OCT) solution, and stored at $-80^{\circ} \mathrm{C}$.

Immunofluorescence. Tissue specimens frozen in OCT solution were sectioned ( $5 \mu \mathrm{m}$ thick), mounted on slides, and air dried for $30 \mathrm{~min}$. The primary antibody was goat anti-mouse CD31 (AF3628; R\&D Systems, Minneapolis, MN, USA), and the secondary antibody was anti-goat Alexa Fluor 546 (Life Technologies, Grand Island, NY, USA). The sections showing immunofluorescence were scanned with a microscope (BZ-9000; Keyence Japan, Osaka, Japan).

Quantification of dextran distribution. Dextran distribution was evaluated by arbitrary selected four hotspots per tumour. These hotspots were selected from the central area of the tumour including cancer cell nests, and avoiding the peritumoral stromal areas. The fluorescent area above a certain threshold in each 200 magnification field was calculated using the Image $J$ software (v1.44p, Rasvand, National Institutes of Health, USA), and the measurement results were averaged.

Tumour treatment study. Mice bearing tumours (two CXs (BxPC-3 and MIA PaCa-2) and three TGs (PC-03, 09, and 10)) received an intra-peritoneal injection of GEM $\left(100 \mathrm{mg} \mathrm{kg}^{-1}\right)$ or saline $(100 \mu \mathrm{l})$ twice a week for 2 weeks. The treatment was combined with intravenous injection of iRGD peptide at $8 \mu \mathrm{mol} \mathrm{kg}^{-1}$ or PBS $10 \mathrm{~min}$ before GEM injection. The mice were weighed and tumour volume was measured twice per week. All xenografts were harvested 28 days after the start of treatment. Tumour volume was calculated using the formula: volume $\left(\mathrm{mm}^{3}\right)=\left(d^{2} \times D\right) / 2$, where $d$ is the smallest and $D$ is the largest tumour diameter.

Clinical specimens of pancreatic cancer. Forty-eight consecutive patients, who underwent surgery for pancreatic cancer at Tsukuba University Hospital between September 2003 and April 2009, were included in the study. Histologically proven cases of pancreatic ductal adenocarcinoma were included, and cancers derived from intraductal papillary mucinous neoplasms were excluded. None of the patients had received neo-adjuvant chemotherapy or irradiation before surgery. Specimens of pancreatic cancer tissue obtained with the patient's consent were fixed in $10 \%$ formalin solution and embedded in paraffin. The pathological diagnoses and classifications were made on the basis of the UICC TNM classification of malignant tumours, 7th edition (Sobin et al, 2009). 
Immunohistochemistry. Paraffin-embedded sections were deparaffinised in xylene and dehydrated through a graded ethanol series. The sections were heated in Tris-EDTA buffer ( $\mathrm{pH} \mathrm{9.0)} \mathrm{for} 10 \mathrm{~min}$ at $121^{\circ} \mathrm{C}$ in an autoclave for antigen retrieval, and endogenous peroxidase was quenched in $0.03 \%$ hydrogen peroxidase for $15 \mathrm{~min}$. The specimens were then incubated with the primary antibody: anti-NRP1 (sc-5307, 1:50; Santa Cruz Biotechnology, Santa Cruz, CA, USA) overnight at $4{ }^{\circ} \mathrm{C}$, or anti-CD31 (LS-B1932; LifeSpan BioSciences, Seattle, WA, USA) for $1 \mathrm{~h}$ at room temperature. The remaining steps were carried out with the Envision + System-HRP (DAKO, Glostrup, Denmark), followed by counterstaining with haematoxylin. Specificity of this antibody was verified by immunostaining of human kidney tissue samples. To assess tissue NRP1 expression, the sections were scored semiquantitatively (on a scale of Immunohistochemistry (IHC)-0-3: negative $=0$, weak $=1$, moderate $=2$, and strong $=3$ ) according to the intensity of chromogen deposition in $10 \%$ or more of the neoplastic cells.

Microvessel density. Microvessel density (MVD) was measured by counting the number of CD31-positive blood vessels at selected
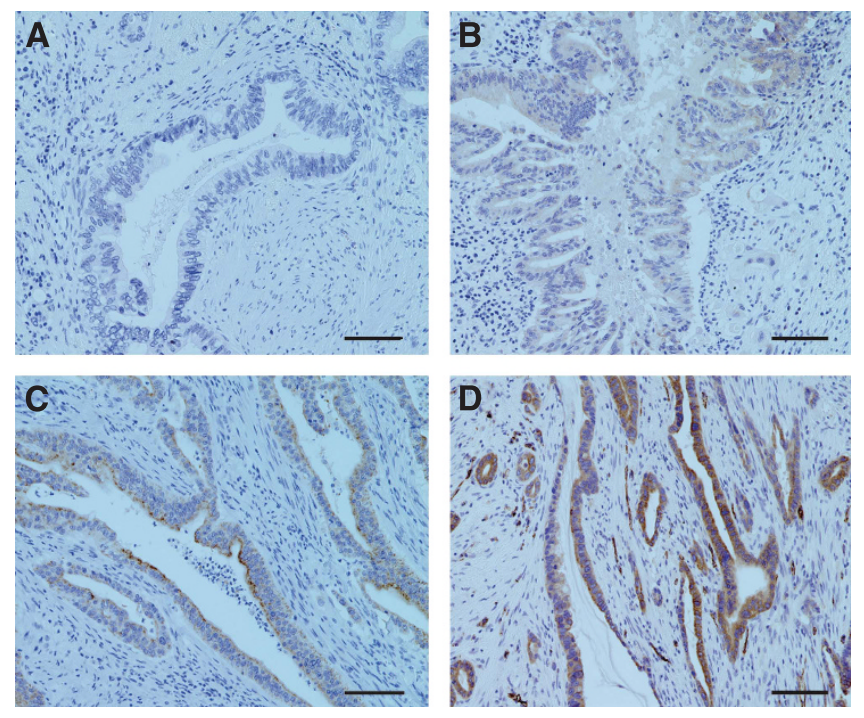

\section{E}

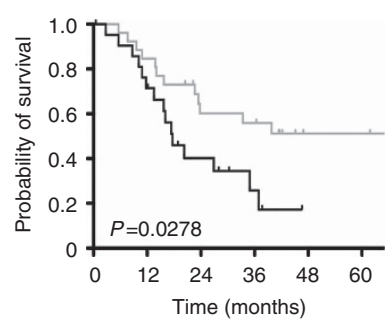

\section{$F$}

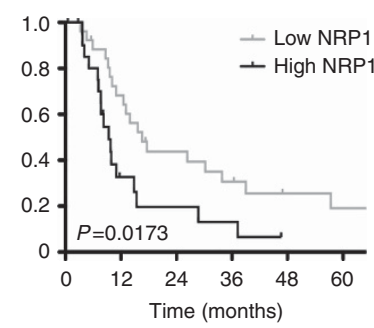

Number at risk

$\begin{array}{lllllll}\text { Low NRP1 } & 26 & 23 & 15 & 14 & 7 & 7\end{array}$

$\begin{array}{lllllll}\text { High NRP1 } & 22 & 16 & 8 & 4 & 0 & 0\end{array}$

Figure 1. (A-D) Representative photomicrographs showing immunohistochemical staining and detailed expression of neuropilin-1 (NRP1, brown colour) in pancreatic ductal adenocarcinomas. Nine (18.8\%) cases were negative (IHC-0, A), 17 (35.4\%) were weakly positive $(\mathrm{IHC}-1+, \mathrm{B}), 12$ (25.0\%) were moderately positive (IHC-2 + , C), and 10 (20.8\%) were strongly positive (IHC-3 + , D). (E and F) Kaplan-Meier analysis of overall survival $(\mathbf{E})$ and disease-free survival $(\mathbf{F})$ in relation to low $(\mathrm{IHC}-0 / 1+)$ or high $(\mathrm{IHC}-2+/ 3+)$ NRP1 expression in pancreatic cancer. Patients with high NRP1 expression showed shorter overall survival $(P=0.0278)$ and disease-free survival $(P=0.0173)$ than patients with low NRP1 expression.

Table 1. Correlation between neuropilin-1 (NRP1) expression and clinicopathological parameters of patients with pancreatic cancer

\begin{tabular}{|l|c|c|c|c|c|}
\cline { 3 - 5 } \multicolumn{5}{|c|}{ NRP1 level } \\
\hline Characteristics & All cases & Low & High & $\chi^{2}$ & P-value \\
\hline Total participants & 48 & 26 & 22 & & \\
\hline
\end{tabular}

\section{Age at surgery (years)}

\begin{tabular}{|l|l|r|r|l|l|}
\hline$<60$ & 14 & 8 & 6 & 0.003 & 0.958 \\
\hline
\end{tabular}

Gender

\begin{tabular}{|l|l|l|l|l|l|}
\hline Male & 24 & 13 & 11 & 0.084 & 0.772 \\
Female & 24 & 13 & 11 & & \\
\hline
\end{tabular}

Tumour grade

\begin{tabular}{|l|r|r|r|l|l|}
\hline Well & 2 & 1 & 1 & 0.031 & 0.985 \\
Moderately & 44 & 24 & 20 & & \\
Poorly & 2 & 1 & 1 & & \\
\hline
\end{tabular}

\section{Tumour location}

\begin{tabular}{|l|r|r|r|l|l|}
\hline Head & 40 & 23 & 17 & 0.420 & 0.517 \\
Body/Tail & 8 & 3 & 5 & & \\
\hline
\end{tabular}

Tumour size

\begin{tabular}{|l|r|r|r|l|l|}
\hline$<40 \mathrm{~mm}$ & 29 & 16 & 13 & 0.015 & 0.902 \\
$\geqslant 40 \mathrm{~mm}$ & 19 & 10 & 9 & & \\
\hline
\end{tabular}

\section{T factor}

\begin{tabular}{|l|r|r|r|r|r|}
\hline $\mathrm{T} 1 / \mathrm{T} 2$ & 2 & 2 & 0 & 1.812 & 0.404 \\
$\mathrm{~T} 3$ & 16 & 8 & 8 & & \\
$\mathrm{~T} 4$ & 30 & 16 & 14 & & \\
\hline
\end{tabular}

Lymph-node metastasis

\begin{tabular}{|l|r|r|r|l|l|}
\hline Positive & 35 & 17 & 18 & 0.904 & 0.342 \\
Negative & 13 & 9 & 4 & & \\
\hline Vascular invasion & $\begin{array}{l}\mid \\
\text { Positive }\end{array} \quad 12$ & 4 & 8 & 1.790 & 0.181 \\
Negative & 36 & 22 & 14 & & \\
\hline
\end{tabular}

Perineural invasion

\begin{tabular}{|c|c|c|c|c|c|}
\hline Positive & 24 & 11 & 13 & 0.755 & 0.385 \\
\hline Negative & 24 & 15 & 9 & & \\
\hline \multicolumn{6}{|c|}{ Microvessel density ${ }^{a}$} \\
\hline$<25$ & 16 & 13 & 3 & 5.549 & 0.018 \\
\hline$\geqslant 25$ & 32 & 13 & 19 & & \\
\hline
\end{tabular}

Resection margin

\begin{tabular}{|l|r|r|r|l|l|}
\hline Positive & 13 & 7 & 6 & 0.089 & 0.765 \\
Negative & 35 & 19 & 16 & & \\
\hline CEA & 20 & 13 & 7 & 0.959 & 0.327 \\
\hline Normal & 28 & 13 & 15 & & \\
High & &
\end{tabular}

\section{CA19-9}

\begin{tabular}{|l|r|r|r|r|l|}
\hline Normal & 16 & 12 & 4 & 3.032 & 0.082 \\
High & 32 & 14 & 18 & & \\
\hline
\end{tabular}

Abbreviation: $C E A=$ carcinoembryonic antigen.

${ }^{\mathrm{a}}$ Number of vessels per 100 magnification field. 
hotspots in high-magnification fields $(\times 200)$. Four randomly selected regions were analysed and averaged.

Statistical analysis. Values are presented as the means and standard deviation. Statistical analyses were conducted using Dr SPSS II for Windows software (SPSS Inc., Chicago, IL, USA). Differences between groups were assessed statistically using Student's $t$-test or Tukey-Kramer test for parametric data, or the Mann-Whitney $U$-test for non-parametric data. Overall and disease-free survival curves were drawn according to the KaplanMeier method, and differences between the curves were analysed by applying the log-rank test. Multivariate Cox regression analysis was used to analyse the independent prognostic factors related to survival. Differences at $P<0.05$ were considered to be statistically significant.

\section{RESULTS}

Correlation of NRP1 overexpression with poor outcome in clinical cases of pancreatic cancer. Neuropilin-1 immunostaining in clinical specimens of pancreatic ductal adenocarcinoma from 48 patients was predominantly identified in the cytoplasm and on the cell surfaces of malignant ductal cells (Figure 1). According to the semi-quantitative assessment criteria employed, the specimens were divided into 4 groups: 9 cases (19.7\%) that were negative (IHC-0, Figure 1A), $17(35.4 \%)$ that were weakly immunostained (IHC-1 + , Figure 1B), 12 (25.0\%) that were moderately immunostained (IHC-2 +, Figure 1C), and 10 (20.8\%) that showed strongly positive immunostaining (IHC-3 + , Figure 1D). Comparisons between low NRP1 expression (IHC-0/1+) and high NRP1 expression (IHC-2+/3+) demonstrated similar clinicopathological features, except for MVD within the tumour $(P=0.018$, Table 1$)$. The 3- and 5-year survival rates for the 48 patients overall were $54.2 \%$ and $12.5 \%$, respectively. Kaplan-Meier analysis demonstrated a significant difference in overall survival between the groups showing high NRP1 expression (median 16.7 months, range 0.5-46.6 months) and low NRP1 expression (median 34.9 months, range 5.6-94.3 months) $(P=0.0278$, Figure 1E). Diseasefree survival also differed significantly between high (median 8.2 months, range 0.5-46.6 months) and low NRP1 expression (median 16.1 months, range 3.2-94.3 months) $\quad(P=0.017$, Figure $1 \mathrm{~F})$. Multivariate analysis using the Cox proportional hazards model indicated that, apart from positivity for lymph-node metastasis (hazard ratio $(\mathrm{HR})=5.620,95 \%$ confidence interval (CI): $1.312-24.071, P=0.020)$, involvement of the resection margin $(\mathrm{HR}=5.394,95 \% \mathrm{CI}: 2.227-13.068, P<0.001)$ and high expression of NRP1 $(\mathrm{HR}=2.391,95 \% \mathrm{CI}: 1.045-5.469, P=0.039)$ were significantly correlated with poor overall survival, and were independent prognostic factors for pancreatic cancer (Table 2).

iRGD peptide facilitates drug penetration into murine pancreatic cancer models showing NRP1 overexpression. To investigate the activity of iRGD peptide, co-injection of Evans blue dye, and fluorochrome-labelled dextran was performed. Evans blue dye accumulation was enhanced 1.9-fold in two CXs (BxPC-3 and MIA $\mathrm{PaCa}-2$ ) by co-administration of iRGD; however, enhanced dye accumulation was not observed in the remaining three CXs (Figure 2A; Supplementary Figure S1).

To evaluate the correlation between the iRGD inducing drug penetration effect and NRP1 expression in cancer cells, IHC was performed. Two CXs that showed enhanced dye accumulation were strongly positive $(3+)$, and the remaining three CXs were weakly positive $(1+)$ (Figure 2B; Supplementary Figure S2).

Three CXs (BxPC-3, MIA PaCa-2, and SUIT-2) and three TGs that showed NRP1 overexpression (Supplementary Figure S2) were employed in dextran experiments. Co-administration of iRGD peptide induced the dextran penetration into tumour parenchyma compared with dextran single administration (Figures 2C and D). Extended dextran-positive areas in CXs were 1.8-fold in BxPC-3 $(P=0.001)$ and 2.1 -fold in MIA PaCa-2 $(P=0.024)$, but had no effect in SUIT-2 (Figure 2E). Penetration of dextran by coadministration of iRGD peptide was also observed in TGs, the areas of dextran distribution were extended 1.7-fold in PC-03 $(P=0.008), 3.0$-fold in PC-09 $(P=0.001)$, and 1.9 -fold in PC-10 $(P=0.040)$ (Figure 2E).

Enhancement of the anticancer effect by co-administration of GEM and iRGD peptide in comparison with GEM monotherapy. Enhanced drug penetration into tumour was evaluated by Evans blue dye as a drug substitute tracer (Figure 3A). Drug accumulations in two CXs and one TG (PC-03) were significantly enhanced by iRGD co-administration; however, the effects in other two TGs (PC-09 and 10) were not remarkable. Similarly, co-administration of iRGD with GEM significantly decreased tumour growth in comparison with GEM monotherapy in BxPC-3 $(P=0.046$, Figures $3 \mathrm{~B}$ and $\mathrm{C})$ and MIA PaCa-2 $(P=0.037$, Figure $3 \mathrm{C})$. One TG model (PC-03) also showed a significant tumour growth

Table 2. Cox proportinal hazards model of prognostic factors in patients with pancreatic cancer $(n=48)$

\begin{tabular}{|c|c|c|c|c|c|c|}
\hline \multirow[b]{2}{*}{ Factor } & \multicolumn{3}{|c|}{ Univariate analysis } & \multicolumn{3}{|c|}{ Multivariate analysis } \\
\hline & HR & $95 \% \mathrm{Cl}$ & $P$-value & HR & $95 \% \mathrm{Cl}$ & $\boldsymbol{P}$-value \\
\hline Age $(\geqslant 60$ years $)$ & 0.934 & $0.418-2.084$ & 0.867 & NA & NA & NA \\
\hline Gender (male) & 0.885 & $0.415-1.887$ & 0.752 & NA & NA & NA \\
\hline $\mathrm{T}$ factor $(\mathrm{T} 4)$ & 1.433 & $0.642-3.199$ & 0.380 & NA & NA & NA \\
\hline Tumour size $(\geqslant 40 \mathrm{~mm})$ & 2.166 & $1.010-4.646$ & 0.047 & 1.738 & $0.748-4.042$ & 0.199 \\
\hline Lymph-node status (positive) & 6.712 & $1.585-28.421$ & 0.010 & 5.620 & $1.312-24.071$ & 0.020 \\
\hline Vascular invasion (positive) & 1.768 & $0.769-4.068$ & 0.180 & NA & NA & NA \\
\hline Perineural invasion (positive) & 1.726 & $0.797-3.740$ & 0.166 & NA & NA & NA \\
\hline Resection margin (positive) & 5.757 & $2.501-13.251$ & $<0.001$ & 5.394 & $2.227-13.068$ & $<0.001$ \\
\hline Microvessel density ${ }^{a}(\geqslant 25)$ & 1.214 & $0.543-2.715$ & 0.637 & NA & NA & NA \\
\hline Neuropilin-1 expression (high) & 2.334 & $1.072-5.083$ & 0.033 & 2.391 & $1.045-5.469$ & 0.039 \\
\hline
\end{tabular}


A
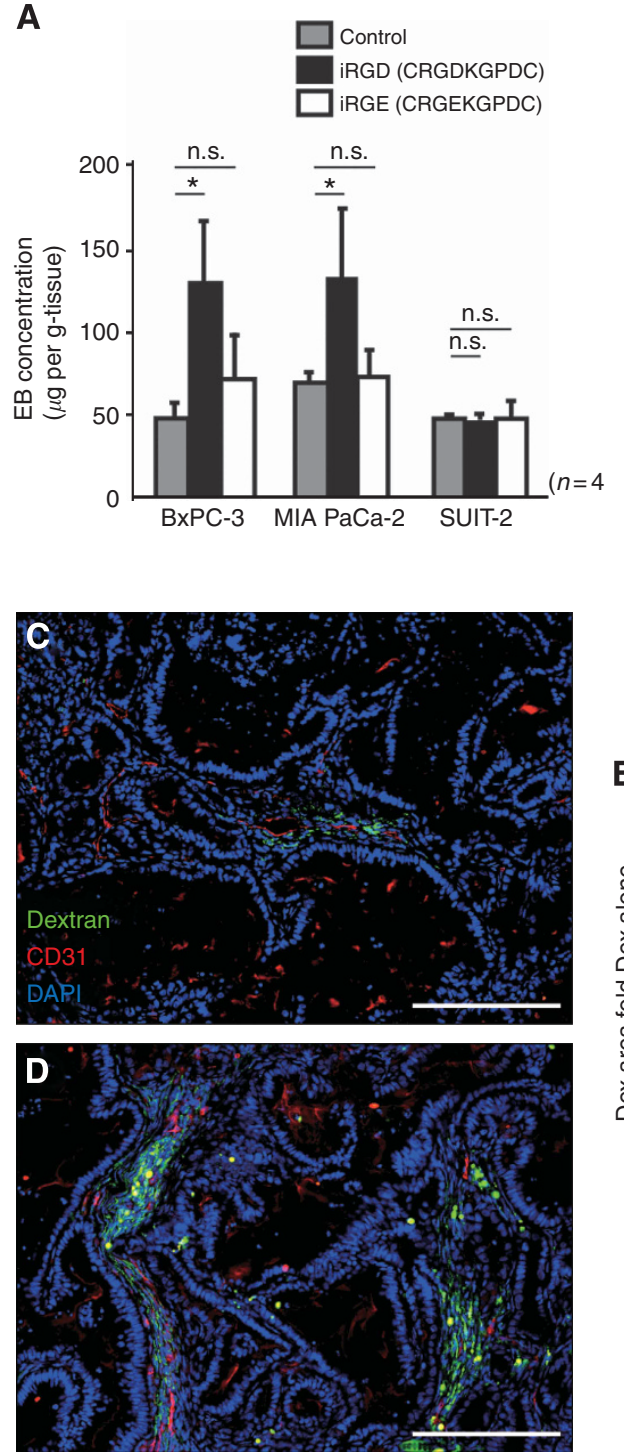

B
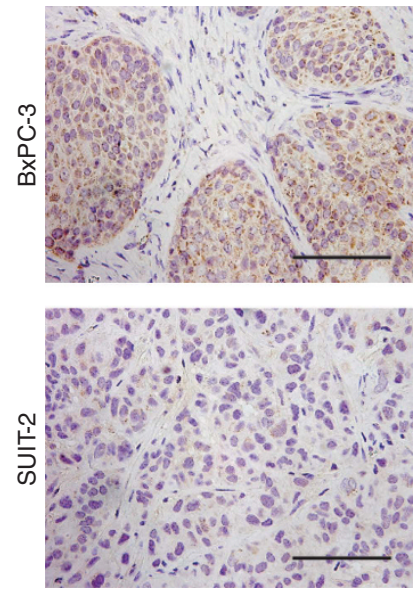
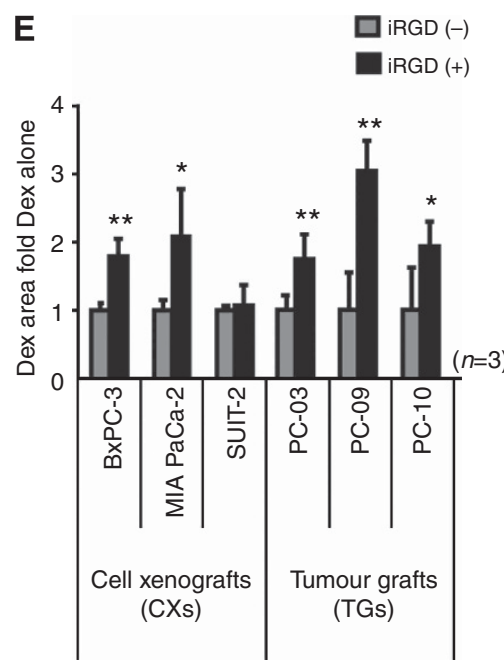

Figure 2. (A) Evans blue dye accumulation assay for cell line-based xenograft models (cell xenografts: CXs). Co-administration of iRGD peptide with a drug (black bar) was compared with administration of a single drug alone (grey bar) or iRGE peptide (white bar). Dye accumulation was enhanced 1.9-fold in BxPC-3 and MIA PaCa-2, but not in SUIT-2. Control peptide (iRGE) showed no enhancement of dye accumulation ( $N=4$ per group). (B) Neuropilin-1 (NRP1) expression in animal models ( $\times 400$ magnification; scale bar, $100 \mu \mathrm{m}$ ). Overexpression of NRP1 was shown in BxPC-3 (upper) and MIA PaCa-2 (not shown), but not in SUIT-2 (lower). (C and D) Distribution of fluorochrome (Alexa-488, green)-labelled dextran in frozen sections of PC-09 ( $\times 200$ magnification; scale bar, $200 \mu \mathrm{m}$ ). Tumour vasculature was stained with anti-CD31 antibody (red), and nuclei were stained with DAPI (blue). Dextran appeared faintly after single administration (C), and it was more widely distributed with a stronger intensity after co-administration with iRGD peptide (D). (E) The areas of dextran distribution were calculated and compared. iRGD co-administration increased the area of dextran distribution 1.8-fold in BxPC-3 $(P=0.001), 2.1$-fold in MIA PaCa-2 $(P=0.024), 1.7$-fold in PC-03 ( $P=0.008), 3.0$-fold in PC-09 $(P=0.001)$, and 1.9-fold in PC-10 $(P=0.040)$. ${ }^{*} P<0.05,{ }^{\star *} P<0.01$.

suppression by the combination therapy $(P=0.048)$, but the other two TGs (PC-09 and 10) exhibited no significant differences (Figure 3C). The body weight of mice was decreased on days 12 and 15 in the GEM administered groups relative to the control group, but there was no significant difference in body weight between the iRGD co-administration group and the GEM monotherapy group (data not shown).

\section{DISCUSSION}

The present study conducted to re-evaluate the use of iRGD peptide demonstrated that it boosted the accumulation of drugs in two of five pancreatic cancer CXs that showed high expression of
NRP1, and the anticancer effects of GEM were also enhanced by iRGD co-administration in these two CXs. We concluded that iRGD co-administration therapy would be indicated for nearly half of all patients with pancreatic cancer showing NRP1 overexpression, and we further evaluated this possibility using clinically relevant murine pancreatic cancer TG models. Enhancement of drug accumulation by iRGD was also observed in TGs, but the effects were less marked than those in CXs. Additionally, a significant anticancer booster effect of GEM plus iRGD combination therapy was observed in only one model.

First, our experiments using five CXs reconfirmed the enhanced drug accumulation effect of iRGD peptide. It was noteworthy that our experiments demonstrated that the effect of iRGD was dependent on the level of NRP1 expression; that is, the effects of iRGD were marked in two high-NRP1 CXs, but not significant in 
A

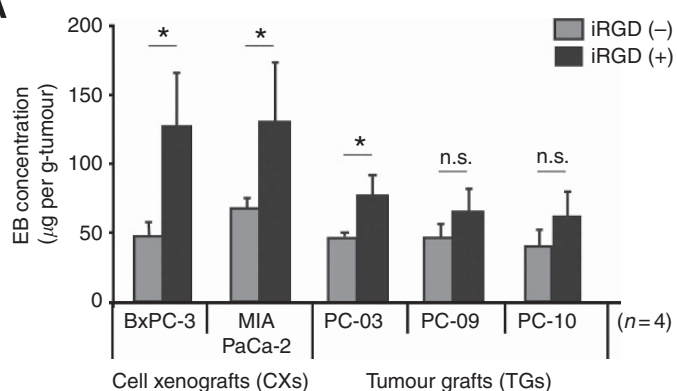

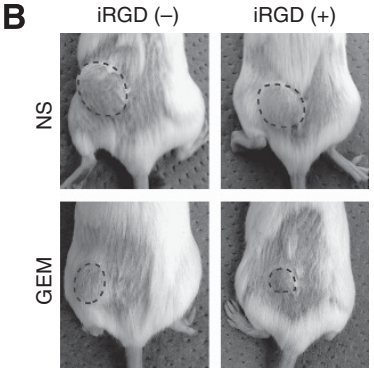
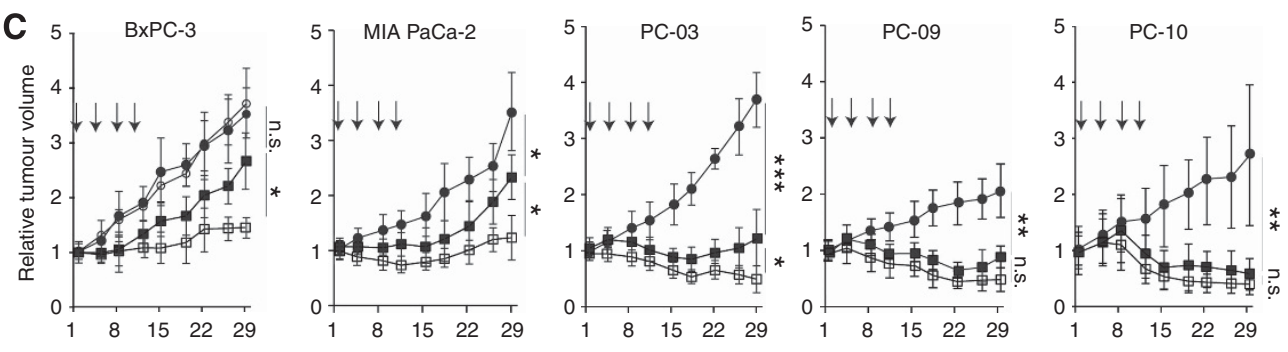

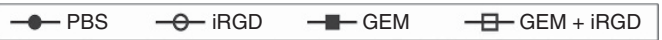

Figure 3. Tumour treatment studies involving co-administration of iRGD peptide. (A) Enhanced drug accumulation into tumour using Evans blue dye as a drug substitute tracer. Drug accumulations were enhanced about twofolds by iRGD in two CXs and one TG; however, the effects were not significant in another two TGs. (B) Tumour appearances in ectopic BxPC3 models at 28 days after the treatment initiation. (C) Tumour growth

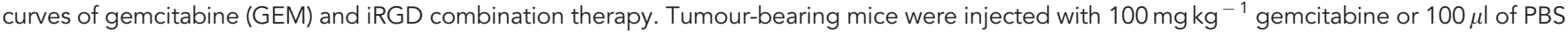
(twice per week for 2 weeks; days 1, 4, 8, and 11; arrows show the day of infusion) combined with injection of PBS (Control, and GEM groups) or $8 \mu \mathrm{mol} \mathrm{kg}{ }^{-1}$ iRGD peptide (iRGD, and GEM + iRGD group) 10 min beforehand ( $N=4$ per group). Tumour growth curves of two CXs (BxPC-3 and MIA PaCa-2) and three TGs (PC-03, PC-09, and PC-10) are indicated. ${ }^{\star} P<0.05,{ }^{\star} P<0.01,{ }^{\star} * \star P<0.001 ;$ n.s., not statistically significant.

three low-NRP1 models. These results suggest that iRGD peptide co-administration would be beneficial for subset patients with NRP1-overexpressing tumours, similarly to a molecular targeting agent.

We then evaluated the populations of pancreatic cancer overexpressing NRP1 in clinical specimens. Neuropilin-1 overexpression was seen in $45.8 \%$ of specimens, and the patients concerned had worse outcomes than patients whose tumours had low NRP1 expression (Figures $1 \mathrm{E}$ and F). Neuropilin-1 is known to be a co-receptor that enhances the binding of VEGF-A to the VEGF receptor (Soker et al, 1998), and NRP1 overexpression induces upregulation of VEGF signalling that is associated with angiogenesis and cancer metastases (Poon et al, 2001). Our finding that NRP1 overexpression was associated with higher MVD (Table 1) was considered to reflect upregulation of VEGF signalling. However, it should also be noted that resistance to GEM might have contributed to the differences in outcome. Neuropilin-1-overexpressing pancreatic cancer cell lines showed chemoresistance to GEM in vitro (Wey et al, 2005). All of the patients included in our study had received GEM-based adjuvant chemotherapy, and the effects of drug resistance should therefore be considered.

The effect of iRGD peptide in enhancing drug penetration is expected to improve the efficacy of treatment for dismal solid tumours, including pancreatic cancer. However, CXs do not adequately represent the clinical features of pancreatic cancer. We therefore employed pancreatic TGs, which have been reported to show better clinical predictive ability (Dong et al, 2010; Hidalgo et al, 2011; Morelli et al, 2012), and our previous evaluation had indicated that the drug delivery characteristics of TGs were more clinically reproducible. Histological findings of pancreatic TGs were more similar to clinical pancreatic cancer compared with CXs, as showing the atypical cancer glands and stromal tissues (Supplementary Figure S2). Internalised-RGD peptide certainly induced drug penetration in all TGs that were overexpressed NRP1, combination therapy using iRGD peptide was considered to be applicable for clinical pancreatic cancer.

Finally, the efficacy of iRGD peptide co-administration with GEM was verified using five pancreatic cancer models that showed enhanced drug accumulation in dextran experiments. In three models, iRGD peptide significantly enhanced drug accumulation into tumour (Figure 3A). Anticancer effect of GEM was also enhanced by iRGD co-administration in these three models (Figure 3C). Therefore, it seems possible to co-administer GEM, which is a key first-line drug for pancreatic cancer, with iRGD peptide, though the molecular size of GEM $(0.3 \mathrm{kDa})$ is smaller than that of agents previously validated $(0.6 \mathrm{kDa}-130 \mathrm{~nm}$, Sugahara et al, 2010). On the other hand, enhanced drug accumulations by iRGD peptide in the remaining two TGs were limited (Figure 3A), and the anticancer effects were also not significantly enhanced (Figure 3C). A major factor influencing the results was considered to be different histological findings among these models. Drug penetrations around the perfusing tumour vessels were not so different between CXs and TGs as shown in dextran experiments (Figure 2E). However, TGs included relative fewer blood vessels and more stromal tissues like clinical pancreatic cancer, as we previously reported (Akashi et al, 2013). We therefore considered that the efficacy of iRGD co-administration might be limited in clinical pancreatic cancer, characterised as poor vascularity and prominent desmoplastic reaction. A TG model (PC-03) that showed significant effect of iRGD peptide was established by transplantation of liver metastatic cancer tissue, whereas the other two TGs (PC-09 and 10) were established from primary pancreatic cancer. Histological findings of PC-03 (Supplementary Figure S2) were relatively similar to CXs, as characterised as hypervascular and fewer stromal tissues (Supplementary Figure S2; Akashi et al, 2013). Paradoxically, it is presumed that the impact of histological features on the iRGD efficacy is greater. Though, the difference of 
drug susceptibility between CXs and TGs should be accounted for the interpretation of our results. As shown in Figure 3C, GEM monotherapy showed $<50 \%$ inhibition of tumour growth in two CXs. In contrast, tumour growths in both TGs were significantly supressed by GEM monotherapy. Therefore, further validations, such as experiments using GEM-resistant TGs, might be required.

Our re-evaluations of iRGD peptide demonstrated a substantial booster accumulation effect of drugs in mouse pancreatic cancer models with high NRP1 expression, and this effect may be exploitable in nearly half of all patients with pancreatic cancer showing high NRP1 expression. Since the booster anticancer effects of iRGD co-administration with GEM were marked only in cell line-based models but not so great in TGs, the possible clinical application of iRGD peptide should be considered carefully.

\section{ACKNOWLEDGEMENTS}

We thank Kazuki N Sugahara, Erkki Ruoslahti, and the members of the Cancer Centre at Sanford-Burnham Medical Research Institute, La Jolla for providing the samples of their peptide. This study was supported by a Grant-in-Aid for Scientific Research (KAKENHI, 23300362 and 23659635) from The Ministry of Education, Culture, Sports, Science, and Technology of Japan.

\section{CONFLICT OF INTEREST}

The authors declare no conflict of interest.

\section{REFERENCES}

Akashi Y, Oda T, Ohara Y, Miyamoto R, Hashimoto S, Enomoto T, Yamada K, Kobayashi A, Fukunaga K, Ohkochi N (2013) Histological advantages of the tumor graft, a murine model involving transplantation of human pancreatic cancer tissue fragments. Pancreas 42: 1275-1282.

Burris 3rd HA, Moore MJ, Andersen J, Green MR, Rothenberg ML, Modiano MR, Cripps MC, Portenoy RK, Storniolo AM, Tarassoff P, Nelson R, Dorr FA, Stephens CD, Von Hoff DD (1997) Improvements in survival and clinical benefit with gemcitabine as first-line therapy for patients with advanced pancreas cancer: a randomized trial. J Clin Oncol 15: 2403-2413.

Dong X, Guan J, English JC, Flint J, Yee J, Evans K, Murray N, Macaulay C, Ng RT, Gout PW, Lam WL, Laskin J, Ling V, Lam S, Wang Y (2010) Patient-derived first generation xenografts of non-small cell lung cancers: promising tools for predicting drug responses for personalized chemotherapy. Clin Cancer Res 16: 1442-1451.

Fukahi K, Fukasawa M, Neufeld G, Itakura J, Korc M (2004) Aberrant expression of neuropilin-1 and -2 in human pancreatic cancer cells. Clin Cancer Res 10: 581-590.

Hansel DE, Wilentz RE, Yeo CJ, Schulick RD, Montgomery E, Maitra A (2004) Expression of neuropilin-1 in high-grade dysplasia, invasive cancer, and metastases of the human gastrointestinal tract. Am J Surg Pathol 28: 347-356.

Heldin CH, Rubin K, Pietras K, Ostman A (2004) High interstitial fluid pressure - an obstacle in cancer therapy. Nat Rev Cancer 4: 806-813.
Hidalgo M, Brunckheimer E, Rajeshkumar NV, Garrido-Laguna I, De Oliveira E, Rubio-Viqueira B, Strawn S, Wick MJ, Martell J, Sidransky D (2011) A pilot clinical study of treatment guided by personalized tumorgrafts in patients with advanced cancer. Mol Cancer Ther 10: 1311-1316.

Jain RK (1999) Transport of molecules, particles, and cell in solid tumors. Annu Rev Biomed Eng 1: 241-263.

Kolodkin AL, Levengood DV, Rowe EG, Tai YT, Giger RJ, Ginty DD (1997) Neuropilin is a semaphorin III receptor. Cell 90: 753-762.

Li M, Yang H, Chai H, Fisher WE, Wang X, Brunicardi FC, Yao Q, Chen C (2004) Pancreatic carcinoma cells express neuropilins and vascular endothelial growth factor, but not vascular endothelial growth factor receptors. Cancer 101: 2341-2350.

Morelli MP, Calvo E, Ordoňez E, Wick MJ, Viqueira BR, Lopez-Casas PP, Bruckheimer E, Calles-Blanco A, Sidransky D, Hidalgo M (2012) Prioritizing phase I treatment options through preclinical testing on personalized tumorgraft. J Clin Oncol 30: e45-e48.

Müller MW, Giese NA, Swiercz JM, Ceyhan GO, Esposito I, Hinz U, Büchler P, Giese T, Büchler MW, Offermanns S, Friess H (2007) Association of axon guidance factor semaphorin $3 \mathrm{~A}$ with poor outcome in pancreatic cancer. Int J Cancer 121: 2421-2433.

Parikh AA, Liu WB, Fan F, Stoeltzing O, Reinmuth N, Bruns CJ, Bucana CD, Evans DB, Ellis LM (2003) Expression and regulation of the novel vascular endothelial growth factor receptor neuropilin-1 by epidermal growth factor in human pancreatic carcinoma. Cancer 98: 720-729.

Poon RT, Fan ST, Wong J (2001) Clinical implications of circulating angiogenic growth factors in cancer patients. J Clin Oncol 19: 1207-1225.

Ruoslahti E, Bhatia SN, Sailor MJ (2010) Targeting of drugs and nanoparticles to tumors. J Cell Biol 188: 759-768.

Sobin LH, Gospodarowicz MK, Wittekind C (2009) TNM Classification of Malignant Tumours. 7th edn. Wiley-Blackwell: Chichester, UK.

Soker S, Fidder H, Neufeld G, Klagsbrun M (1996) Characterization of novel vascular endothelial growth factor (VEGF) receptors on tumor cells that bind VEGF165 via its exon 7-encoded domain. J Biol Chem 27: 5761-5767.

Soker S, Takashima S, Miao HQ, Neufeld G, Klagsbrun M (1998) Neuropilin1 is expressed by endothelial and tumor cells as an isoform-specific receptor for vascular endothelial growth factor. Cell 92: 735-745.

Sugahara KN, Teesalu T, Karmali PP, Kotamraju VR, Agemy L, Girard OM, Hanahan D, Mattrey RF, Ruoslahti E (2009) Tissue-penetrating delivery of compounds and nanoparticles into tumors. Cancer Cell 16: 510-520.

Sugahara KN, Teesalu T, Karmali PP, Kotamraju VR, Agemy L, Greenwald DR, Ruoslahti E (2010) Coadministration of a tumor-penetrating peptide enhances the efficacy of cancer drugs. Science 328: 1031-1035.

Teesalu T, Sugahara KN, Kotamraju VR, Ruoslahti E (2009) C-end rule peptides mediate neuropilin-1-dependent cell, vascular, and tissue penetration. Proc Natl Acad Sci USA 106: 16157-16162.

Tempero M, Plunkett W, Ruiz Van Haperen V, Hainsworth J, Hochster H, Lenzi R, Abbruzzese J (2003) Randomized phase II comparison of doseintense gemcitabine: thirty-minute infusion and fixed dose rate infusion in patients with pancreatic adenocarcinoma. J Clin Oncol 21: 3402-3408.

Wey JS, Gray MJ, Fan F, Belcheva A, McCarty MF, Stoeltzing O, Somcio R, Liu W, Evans DB, Klagsbrun M, Gallick GE, Ellis LM (2005) Overexpression of neuropilin-1 promotes constitutive MAPK signalling and chemoresistance in pancreatic cancer cells. Br J Cancer 93: 233-241.

This work is published under the standard license to publish agreement. After 12 months the work will become freely available and the license terms will switch to a Creative Commons AttributionNonCommercial-Share Alike 3.0 Unported License.

Supplementary Information accompanies this paper on British Journal of Cancer website (http://www.nature.com/bjc) 